Rapid Reviews COVID-19

\title{
Review 4: "Repurposed antiviral drugs for COVID- 19; interim WHO SOLIDARITY trial results"
}

Emily Walsh ${ }^{1}$

${ }^{1}$ Consultant, Kaiser Permanente, Care Management Institute, USA

Published on: Nov 16, 2020

DOI: $10.1162 / 2 \mathrm{e} 3983 f 5.56 \mathrm{~d} 41668$

License: Creative Commons Attribution 4.0 International License (CC-BY 4.0). 


\section{$\underline{\text { RR:C19 Evidence Scale rating by reviewer: }}$}

- Potentially informative. The main claims made are not strongly justified by the methods and data, but may yield some insight. The results and conclusions of the study may resemble those from the hypothetical ideal study, but there is substantial room for doubt. Decision-makers should consider this evidence only with a thorough understanding of its weaknesses, alongside other evidence and theory. Decisionmakers should not consider this actionable, unless the weaknesses are clearly understood and there is other theory and evidence to further support it.

******************************************

\section{Review:}

The SOLIDARITY Therapeutics Trial ${ }^{1}$, coordinated by the WHO, is the largest randomized trial of pharmaceuticals for management of COVID-19 in hospitalized patients. Presently, 405 hospital sites across 30 countries are participating in the trial, with 11,266 adult patients randomized so far. The design of the trial is "adaptive"meaning that study drugs may be dropped if they are determined to be ineffective or harmful, and new study drugs may be added. To improve feasibility for participating sites, very limited data reporting was required and the study does not stipulate requirements for management of patients randomized to "standard of care" or for supportive care to be provided in addition to pharmaceutical agents for patients randomized to any group. Thus, while not reported in interim results, there was likely considerable variation across participating sites in management of patients that could influence key study outcomes (mortality, disease progression, length of hospital stay).

Key limitations of this study include:

- Variation in control condition. Likelihood of substantial variation in locally defined "standard of care" as the control condition across study sites.

- Variation and uncertainty in other assigned treatments. Lack of information about other supportive care provided to hospitalized patients. Given that this study was performed across hundreds of hospitals in 30 countries, there was likely significant variation in the other supportive interventions that participants received while in hospital that were not tracked or reported in the study. 
- Symptoms of participants not reported or tracked. The majority of participants ( $\sim 60 \%)$ were randomized on day 0-1 of hospitalization, but duration of infection and associated symptoms were not reported or tracked.

- Disease severity is not clearly categorized or described. The report indicates the proportion of patients on ventilation when treatment is initiated (a sub-group analysis is performed for 28-mortality by ventilated vs. not ventilated participants who received remdesivir). However, baseline disease severity is not reported. It is possible that the distribution of participants with differing disease severity at baseline was uneven.

- Variation in available study drugs by study site. Participants in any given study hospital were randomized in equal proportion to control and whichever study drugs were locally available. Reporting of which drugs were available at specific study sites/participating countries is not included in the publication. The availability of all four study drugs may not be equally distributed across sites.

Diagnostic confirmation of SARS-CoV-2 infection was not required. It is not clear that the distribution of those with diagnostic confirmation of infection is equal across groups as this is not required or reported in the publication.

\section{How does evidence from this trial fit with existing evidence?}

The available results for mortality risk from the SOLIDARITY trial are in accordance with the existing understanding of remdesivir for COVID-19. However, the SOLIDARITY trial reports no benefit of remdesivir for disease progression and length of hospital stay. In contrast, other evidence suggests that remdesivir may significantly reduce disease progression.

For example, a rapid response brief published in June 2020 for Alberta Health Services includes evidence from the phase III NIAID Adaptive COVID-19 Treatment Trial

(ACTT). ${ }^{2}$ Interim results from ACTT demonstrated a benefit of remdesivir therapy over placebo for time to recovery:

- Median time to recovery: remdesivir was 11 days (95\% CI: 9-12) vs. placebo was 15 days (95\% CI: 13-19); $\mathrm{p}<0.0001$

\section{References:}


1. Pan H, Peto R, Karim QA, et al. Repurposed antiviral drugs for COVID-19 interim WHO SOLIDARITY trial results. medRxiv. 2020:2020.2010.2015.20209817.

2. Kania-Richmond A, al. e. COVID-19 Scientific Advisory Group Rapid Response Brief - Key Research Question: What is the evidence for effectiveness of remdesivir as a treatment for COVID-19 disease? 2020;

https://www.albertahealthservices.ca/assets/info/ppih/if-ppih-covid-19-sag-effectivenessof-remdesivir-rapid-review.pdf. 